Rochester Institute of Technology

RIT Scholar Works

Presentations and other scholarship

Faculty \& Staff Scholarship

Spring 4-24-2020

\title{
Smart Cities at Play: Lived Experiences, Emerging Forms of Playfulness, and Problems of Participation
}

\author{
Konstantinos Papangelis \\ Rochester Institute of Technology \\ Jin-Ha Lee \\ University of Washington \\ Michael Saker \\ City, University of London \\ Catherine Jones \\ Luxembourg University
}

Follow this and additional works at: https://scholarworks.rit.edu/other

Part of the Other Computer Sciences Commons

\section{Recommended Citation \\ Papangelis K, Saker M, Jones C, Lee J-H. Smart Cities at Play: Lived Experiences, Emerging Forms of Playfulness, and Problems of Participation. Workshop. 30th ACM Human Factors in Computing Systems conference (CHI '20). ISBN: 978-1-4503-6819-3/20/04. Doi: https://doi.org/10.1145/3334480.3375146.}

This Conference Paper is brought to you for free and open access by the Faculty \& Staff Scholarship at RIT Scholar Works. It has been accepted for inclusion in Presentations and other scholarship by an authorized administrator of RIT Scholar Works. For more information, please contact ritscholarworks@rit.edu. 


\section{Smart Cities at Play: Lived Experiences, Emerging Forms of Playfulness, and Problems of Participation}

Konstantinos Papangelis Rochester Institute of Technology

United States of America kxpigm@rit.edu
Jin Ha Lee

University of Washington

United States of America

jinhalee@uw.edu
Michael Saker

City, University of London

United Kingdom

Michael.Saker@city.ac.uk
Catherine Jones

Luxembourg University.

Luxembourg

Catherine.jones@uni.lu

Permission to make digital or hard copies of part or all of this work for personal or

\begin{abstract}
In recent years, the notion of smart cities has become the focus of a growing body of research. To date, much of this attention has revolved around the technical aspect, with related concerns including the creation and implementation of suitable smart city technologies. What is notably missing from these discussions, however, is a consideration of the lived experience of supposedly 'smart spaces' and the extent to which physical and digital environments are currently producing new forms of play and playfulness that can be contextualized within this field. With this in mind, the purpose of our workshop is as follows. First, to provide a platform for researchers and practitioners to engage with these issues that often remain hidden when discussions solely focus on technology. Second, to develop a draft research agenda for challenges that will serve as a primer for future studies examining the topic.
\end{abstract}

\section{Author Keywords}

Smart City; Urban Play; Inclusiveness

\section{CCS Concepts}

-Human-centered computing $\rightarrow$ Human computer interaction $(\mathrm{HCl})$;

\section{NOTE}

This is a pre-print of our CHI'2020 article. 
Download the latest version of the article here: https://doi. org/10.1145/3334480.3375146. The article will be available a a couple of weeks before the $\mathrm{CHI}$ (https://chi2020.acm.org/) conference in April 2020.

Cite as:

Papangelis K, Saker M, Jones C, Lee J-H. Smart Cities at Play: Lived Experiences, Emerging Forms of Playfulness, and Problems of Participation. Workshop. 30th ACM Human Factors in Computing Systems conference $(\mathrm{CHI}$ '20). ISBN: 978-1-4503-6819-3/20/04. Doi: https://doi.org/10.1145/3334480.3375146.

\section{Introduction and Motivation}

Smart cities have been be described as 'places where information technology is combined with infrastructure, architecture, everyday objects, and even our bodies to address social, economic, and environmental problems' [10]. In recent years, this notion of smart cities has become the focus of a growing body of research. An important part of this development has been digital technologies embedded with the global positioning system (GPS). These technologies, and the data they produce, have enabled new forms of urban analysis, urban planning, and the production of more efficient spaces [5]. Locative data, for instance, is regularly used to determine the severity of traffic and predict the arrival of mass transit vehicles, just as it has become pivotal to ridesharing services and the mobile technologies underpinning these applications [2] . locative technologies can also be used to better understand human mobilities, monitor crowds during large gatherings, and theorise the spatial behaviours of tourists [1]. At the same time, GPS enabled smartphones have led to the development of locative media, location-based social networks (LBSN), and emerging forms of pervasive play [7]. In all of these instances, then, digital technologies are increasingly part of our daily lives, just as these technologies are progressively providing the provision for both 'smarter' and more playful spaces.

While this might be the case, "[in] the rush to create socalled 'smart cities' ... much of the attention has been on how to technically create and implement suitable smart city technology" [6]. What is notably missing from these discussions, therefore, is a deeper engagement with the lived experience of supposedly 'smart spaces' that explores the extent to which these physical and digital environments are currently producing new forms of play and playfulness that can be contextualised within this field [9].

It is our contention that the lived experience of smart cities enable both explicit and implicit forms of play. Locative games like Pokèmon GO, for example, explicitly allow users to playfully interact with their environment; forging new social connections, and altering how users feel about themselves and their environment. At the same time, digital technologies configure new approaches to space and place that are comparably playful, but not necessarily planned for. Certainly, the social value of emerging ridesharing services go beyond questions of mobility, permitting novel notions of transportation that potentially provide more poetic ways of traversing the urban environment[4]. Similarly, online hospitality services, such as Airbnb, challenge how short-term accommodation is understood; moving towards a mode of inhabitancy that is perhaps more lived, fluid, and experiential than hotels. However, recent reports have demonstrated that not everyone gets to partake in these services. Numerous stories have emerged about Airbnb, and individuals canceling reservations because of the race of their occupants. Even games like Pokèmon GO have proved problematic, with some players expressing concerns about 
their safety based on their ethnicity, gender, age, and other factors, which can limit their ability to play and can lead to feelings of exclusion [3].

Whether explicit or implicit, then, smart cities are evidently producing both planned and unplanned forms of play that are not immediately accounted for in the context of 'efficiency', but are important for researchers within the field nonetheless. Only by addressing the lived experience of smart cities can we reveal how these spaces are playfully experienced and the impact this can have on both players and society. Importantly, these experiences often remain hidden when discussions solely focus on technology.

\section{Goals, scope, and topics}

The overarching principle of our workshop is to provide a platform to exchange information and experiences, to stimulate discussion, and to explore issues that attend to (1) explicit and implicit forms of play facilitated by the smart city, and (2) how lived experiences of the smart city are currently changing, creating new playful possibilities, as well as moments of resistance, while revealing socio-cultural problems of participation that transcend the technological aspect and often remain hidden from related discussions.

Our main objectives are as follows. First, to articulate a set of specific research challenges around this topic. Second, to produce a draft research agenda that will serve as a primer for future studies examining the topic. Third, to formalize a set of short and long term objectives that will deliver on this agenda.

We aim to solicit involvement from multiple disciplines, including, but not limited to, computer science, information science, media studies, sociology, geography. It is our contention that a plurality of voices will add meaningful con- tours to the varied challenges that currently surround this subject area.

For the workshop we will solicit different types of contributions including research papers, position papers, commentaries, critiques, and demonstrations under the following broad themes:

- The changing perceptions of smart cities in the context of human experience.

- Emerging smart city technologies, such Augmented Reality (AR) and Virtual reality (VR), $5 \mathrm{~g}$, wearable technologies and locative media, and their impact on the lived experience.

- Emerging locative applications and new forms of pervasive play.

- Ridesharing, novel mobilities, and emerging forms of ambulation.

- Laissez faire versus planned play in the city

- Techno-socio-cultural problems of participation that often remain hidden from discussions of smart cities.

- Methods for researching implicit and explicit form of play in a smart city context.

- Related ethical implications for designers and players of smart city play experiences.

With the help of an international program committee, we will select submissions based on a number of criteria, including originality or controversy of the paper, the complementary nature of the authors' research background, and the quality of the authors' previous work related to the workshop topic.

\section{Pre-workshop plans}

Research relating to smart cities and surrounding notions of play has appeared in various $\mathrm{HCl}$ conferences such as 
$\mathrm{CHI}$, MobileHCI, CHIplay, and UbiComp. We therefore intend to spread our call for participation (see page 8) across these communities. We will do so through appropriate mailing lists, and social media channels, such as Twitter and Facebook. Also, the call for participation and materials (e.g. accepted papers, bio of participants) that are related to the workshop will be posted to the workshopâĂŹs website, which is described in more detail below.

With respect to involving practitioners in this workshop, we will draw upon our connections with developers at organizations such as Microsoft, Google, and Niantic Labs, several of whom regularly attend $\mathrm{CHI}$.

Further, to engage with participants that do not traditionally attend $\mathrm{HCl}$ conferences we will leverage our contacts and invite developers, digital media organizations, memory institutions, policy makers, designers, and others who may be interested.

Based on previous experiences, we will have between 15 to 20 attendees with multidisciplinary backgrounds participating in the workshop.

Once we have a list of accepted submissions, we will add them to the website. To better understand each participant's work, we ask them to upload a brief bio and profile picture, alongside their goals, interests, and a blurb detailing their work to the website. In addition, for effective communication with the participants we will invite them to an existing work space on Slack (https://citiesatplay.slack.com).

\section{Workshop structure}

The first part of the workshop will involve participants presenting their projects, research, and concomitant positionss. Presentations will last approximately 5 minute and will adopt the Pecha Kucha format. Depending on the num- ber of submissions, we plan to have between 2 and 4 thematically grouped presentation sessions in the morning. Each round will conclude with a short wrap-up discussion that seeks a common ground for afternoon discussions.

The morning session will also allow time for invited talks by selected experts from research communities and industry.

After a coffee break, demo sessions will take place. Here, participants will present different prototypes including sketches, case studies, and implementations.

The morning session will conclude with a joint lunch. This will allow participants the space to informally discuss their respective presentations, projects and positions in a relaxed atmosphere.

The second part of the workshop will involve a speculative design session, in which groups of 3 to 5 participants will work on specific ideas for future inclusive playful smart cities. To support creativity and originality we will use 2 playful and aleatory methods: (1) 'the thing from the future' method [8] and (2) Mark Wetzel's ideation cards for designing Mixed Reality Games [11].

After a coffee break, each group will pitch their project, and discuss the potential impact of each concept. This will lead into a larger group discussion about relevant ideas, concepts, and issues.

As a final activity, we will plan the next steps in a brainstorming session based on the agenda developed during the workshop. The workshop will then conclude with a brief summary of discussions.

Following the workshop, we will hold a joint dinner to provide participants with the space to further socialize and continue discussions and networking. 
A summary of the preliminary workshop schedule is presented below.

09:00 - 09:15 Opening and Introductions $15 \mathrm{~min}$.

09:15 - 10:45 Participants' Presentations $90 \mathrm{~min}$.

10:45 - 11:15 Coffee break $30 \mathrm{~min}$.

11:15 - 12:00 Demos and hands-on experiences $45 \mathrm{~min}$

12:00 - 13:30 Lunch $90 \mathrm{~min}$

13:30 - 15:00 Group work $90 \mathrm{~min}$

15:00 - 15:30 Coffee break $30 \mathrm{~min}$

15:30 - 16:30 Group results $60 \mathrm{~min}$

16:30 - 17:30 Future work agenda 60 min

17:30 - 17:45 Workshop wrap-up and closing $15 \mathrm{~min}$

\section{Postworkshop plans}

After the workshop, we will:

- Summarize the outcomes and share them with all presented materials on our website.

- Submit a research paper distilling research-specific content from the workshop in a journal or conference.

- Invite the participants to collaborate on a special journal issue on the topic of the workshop.

We will also use this workshop as a touching point for the $\mathrm{HCl}$ urban play community. We plan on doing the following:

- Continue to grow our website with examples from the work of the participants and by continually adding resources (designs, etc.).

- Continue our discussion and communication with the workshop participants through the Cities at Play Slack channel and the location-based games mailing list we recently established - https://www.jiscmail.ac.uk/ LOCATIONBASEDGAMES.
- Use the insights gained from this workshop to inform the design of other activities (e.g. panel, dagstuhl type seminar, etc.) at other venues and conferences to foster further collaboration, enlarge the community, and progress the topic of urban play in the context of smart cities.

\section{Website}

The workshop's website can be found at https://smartcitiesatplay. wordpress.com. Currently, the website contains the call for papers and information about the organizers of the workshop. We will primarily use the website to (1) advertise the workshop, communicate information to the participants, and host information about the participants, and (2) disseminate the workshop outcomes, including all presented materials.

\section{Organizers}

Konstantinos Papangelis Dr. Papangelis is an Assistant Professor at the School of Interactive Games and Media of Rochester Institute of Technology. Dr. Papangelis did his M.Sc, in Human-Computer Interaction at Lancaster University (UK), and his Ph.D. in Computer Science at the University of Aberdeen (UK). He is a fellow of The Royal Society for the Encouragement of Arts (RSA), and is heavily involved with the Special Interest Group on HumanComputer Interaction (SIGCHI) of the Association for Computing Machinery (ACM). Currently, his research focuses on, location-based games and social networks, the physical web, location-based and in-situ crowdsourcing, proximity technologies, extended reality, and multi-sensory entertainment technologies. He has published his work in multiple journals and conferences, such as ACM Transactions on Computer-Human Interaction (TOCHI), and Human Factors in Computing Systems (CHI). He is co-editor and author of two books - 'Smart Cities at Play: Technology and Emerging Forms of Playfulness' (Smart City Series of Else- 
vier; forthcoming) and 'Macro-Task Crowdsourcing: Engaging the Crowds to Address Complex Problems' (Human-

Computer Interaction Series of Springer; ISBN: 978-3030-12334-5). His work has so far been supported by the European Union, The Engineering and Physical Sciences Research Council (EPSRC UK), and the ACM.

Michel Saker Dr. Saker is a Senior Lecturer at City, University of London. $\mathrm{He}$ is also a Visiting Research Fellow at the Web Science Institute at the University of Southampton, and a Convenor for the British Sociological Association (BSA) Digital Sociology Study Group. His research examines digital media technologies and digital cultures along two interconnected lines of enquiry. First, he is interested in the physical, spatial and social consequences of emerging mobile media in urban environments. He is particularly interested in the role locative applications might play in placemaking practices and perceptions of urban space. Second, he is fascinated by the broader impact digital media technologies are having on society. More specifically, he specialises in theories of digitality, often focusing on locative applications, their role in place-making practices and the mediation of daily life. He is co-author of Location-Based Social Media, Space, Time and Identity (Palgrave Macmillan, 2017) and co-editor of Smart Cities at Play: Technology and Emerging Forms of Playfulness (Smart City Series of Elsevier; forthcoming). His research has been published in journals including, New Media and Society, Media Culture and Society, Mobile Media Communications, and First Monday. He has co-edited special issues on locative media and the presentation of the self, as well as smart cities and new forms of playful engagement. He has presented his work at numerous conferences, including the International Communication Association (ICA), British Sociological Association (BSA), and the Media, Communication and Cultural Studies Association (MeCCSA). He has also been in- vited to present his research at a number of industry-focued events.

Catherine Jones $\mathrm{Dr}$ Jones is a Researcher at the University of Luxembourg. Her interests focus on mixed methods geographer/GIS with expertise in social and urban change with a desire to understand the lived city and to develop tools that aid public understanding. She is a fellow of the Royal Geographical Society (RGS). Her expertise includes: (1) modelling health inequalities by developing geodemographic measures (2) To understand past city landscapes she has developed different geovisualization tools for exploration of multimedia data (including Instagram posts, historical records etc) using $\mathrm{HCl}$ practices to develop novel web and AR mobile Apps and (3) the use of geolocated games for cultural heritage access and the role of the city as a playground. She uses qualitative and quantitative methods to evaluate geographic and historic thinking processes to explore interactions between people, places and the lived city. She is published in journals including: Transactions In Human Geography, International journal of Geographical Information Systems, Urban Morphology and $\mathrm{CHI}$.

Jin Ha Lee Dr. Lee is an Associate Professor and the Founder and Director of the GAMER (GAME Research) Group at the University of Washington Information School. She holds an M.S. (2002) and a Ph.D. (2008) from the University of Illinois at Urbana-Champaign. Her research focuses on exploring new ideas and approaches for organizing and providing access to music, multimedia, and interactive media, understanding user behavior related to the creation and consumption of these media, and using these media for informal learning in venues such as libraries and museums. Recently, her research has focused on investigating the impact of location-based augmented reality games and virtual reality games to player behavior, in particular, group 
behavior, intergenerational joint media engagement, and learning. Her work was published in venues across multiple domains, such as Journal of the American Society for Information Science and Technology, Journal of Documentation, Journal of Intelligent Information Systems, Games and Culture, Information Research, and conferences such as ACM Conference on Human Factors in Computing Systems, International Society for Music Information Retrieval, iConference, International Conference on Computer Supported Collaborative Learning, and ACM/IEEE Joint Conference on Digital Libraries. Her research has been funded by the Institute of Museum and Library Services, Washington State Library, Online Computer Library Center, Association for Library and Information Science Education, Korea Electronics Technology Institute, and Epic Games. She serves on the Editorial Board of the Journal of the Association for Information Science and Technology, and Transactions of the International Society for Music Information Retrieval.

\section{REFERENCES}

[1] Syed Ahson, Mohammad llyas, and an O'Reilly Media Company Safari. 2010. Location-Based Services Handbook. OCLC: 1105764861.

[2] Michael Batty. 2012. Smart Cities, Big Data. Environment and Planning B: Planning and Design 39 , 2 (April 2012), 191-193. DOI:

http://dx.doi.org/10.1068/b3902ed

[3] Arpita Bhattacharya, Travis W. Windleharth,

Rio Anthony Ishii, Ivy M. Acevedo, Cecilia R. Aragon, Julie A. Kientz, Jason C. Yip, and Jin Ha Lee. 2019. Group Interactions in Location-Based Gaming: A Case Study of Raiding in PokéMon GO. In Proceedings of the 2019 CHI Conference on Human Factors in Computing Systems (CHI '19). ACM, New York, NY,
USA, Article 587, 12 pages. DOI :

http://dx.doi.org/10.1145/3290605.3300817

[4] Alan Chamberlain, Mads Bødker, and Konstantinos Papangelis. 2017. Mapping Media and Meaning: Autoethnography As an Approach to Designing Personal Heritage Soundscapes. In Proceedings of the 12th International Audio Mostly Conference on Augmented and Participatory Sound and Music Experiences (AM '17). ACM, New York, NY, USA, Article 32, 4 pages. DOI : http://dx.doi.org/10.1145/3123514.3123536

[5] Stephen Goldsmith and Susan Crawford. 2014. The responsive city: engaging communities through data-smart governance. Jossey-Bass, San Francisco, $\mathrm{CA}$

[6] Rob Kitchin. 2018. The realtimeness of smart cities. TECNOSCIENZA: Italian Journal of Science \& Technology Studies 8, 2 (2018), 19-42.

[7] Dale Leorke. 2019. Location-Based Gaming: Play in Public Space. Springer Singapore, Singapore. DOI : http://dx.doi .org/10.1007/978-981-13-0683-9

[8] Riel Miller. 2018. Transforming the Future (Open Access): Anticipation in the 21st Century. Routledge. DOI : http://dx.doi.org/10.4324/9781351048002

[9] Konstantinos Papangelis, Melvin Metzger, Yiyang Sheng, Hai-Ning Liang, Alan Chamberlain, and Vassilis-Javed Khan. 2017. "Get Off My Lawn!": Starting to Understand Territoriality in Location Based Mobile Games. In CHI EA '17 Proceedings of the 2017 CHI Conference Extended Abstracts on Human Factors in Computing Systems. ACM Press, 1955-1961. DOI : http://dx.doi.org/10.1145/3027063.3053154 
[10] Anthony M. Townsend. 2013. Smart cities: big data, civic hackers, and the quest for a new utopia (first edition ed.). W.W. Norton \& Company, New York.

[11] Richard Wetzel, Rod McCall, Anne-Kathrin Braun, and Wolfgang Broll. 2008. Guidelines for designing

augmented reality games. In Proceedings of the 2008 Conference on Future Play Research, Play, Share -

Future Play '08. ACM Press, Toronto, Ontario, Canada, 173. DOI :

http://dx.doi.org/10.1145/1496984.1497013 


\section{Call for Papers}

\section{Smart Cities at Play: Lived Experiences, Emerging Forms of Playfulness, and Problems of Participation}

\section{Call for Participation}

Smart cities can be described as places where physical environments are combined with information technology to rectify socioeconomic and environmental issues. While this might be the case, for the most part scholarly attention has focused on the technologies used to construct these environments. What is missing from these discussions is a deeper engagement with the lived experience of 'smart spaces', and the extent to which the confluence of the physical and digital is currently configuring new explicit and/or implicit forms of play. To this end, our workshop will explore how experiences of the city might be changing as a result of new technological practices that are currently creating both explicit and implicit playful possibilities, as well as moments of resistance that reveal socio-cultural problems of participation.

We invite researchers and practitioners from all related disciplines to contribute 4 to 6 page papers that reflect on the following topics:

- The changing perceptions of smart cities in the context of human experience.

- Emerging smart city technologies, such AugmentedReality (AR) and Virtual reality (VR), 5g, wearable technologies and locative media, and their impact onthe lived experience.

- Emerging locative applications and new forms of pervasive play.

- Ridesharing, novel mobilities, and emerging forms of ambulation

- Laissez faire versus planned play in the city

- Techno-socio-cultural problems of participation that often remain hidden from discussions of smart cities.

- Methods for researching implicit and explicit forms of play in a smart city context.

- Related ethical implications for designers and playersof smart city play experiences.

Aside from papers, we invite demonstrations of prototypes and completed projects.

Please submit your paper through the EasyChair chair system: https://easychair.org/conferences/?conf=cap2020.

We will select submissions based on a number of criteria. This includes originality or controversy of the paper, the complementary nature of the authors' research background, and the quality of the authors' previous work related to the workshop topic. At least one author of each accepted paper must attend the workshop and all workshop participants must register not only for the workshop but also for at least one day of the conference.

For more information about the workshop either visit https://smartcitiesatplay.wordpress.com or email the organizers at Kpapangelis@me.com (CC Michael.Saker@city.ac.uk, Catherine.Jones@uni.lu,jinhalee@uw.edu) 